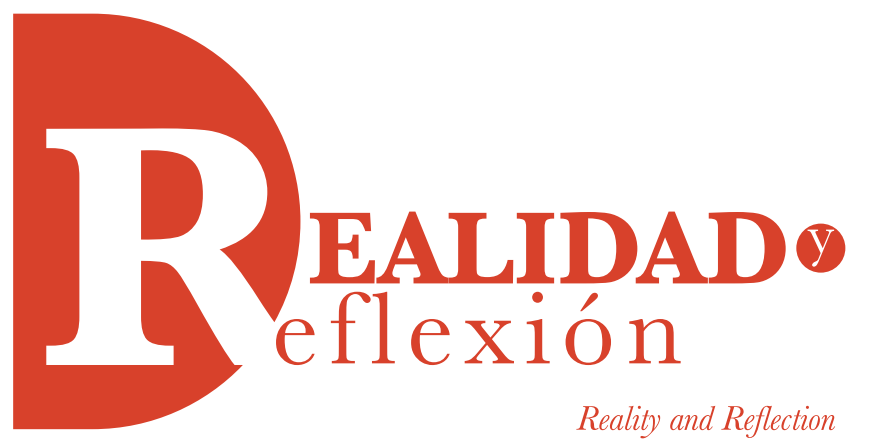

ISSN 1992-6510

e-ISSN 2520-92990

Año 17, N 45, San Salvador, El Salvador, Centroamérica. Revista Semestral Enero-Junio 2017

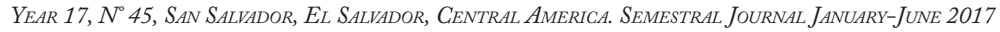

\title{
Educación, investigación e innovación y sus perspectivas desde el mundo laboral. Casos de las universidades salvadoreñas
}

\section{Education, research and innovation and their perspectives about working world. Salvadorans universities cases}

\author{
Roberto Carbajal \\ Docente Investigador Facultad de Ciencias Sociales Universidad Francisco Gavidia (UFG) \\ Master en Métodos de Investigación Social Universidad de El Salvador (UES) \\ Licenciado en Educación - Idioma Inglés (UES)
}

Fecha de recepción: 2 de enero de 2017 Fecha de aceptación: 4 de mayo de 2017

\section{RESUMEN}

El presente artículo se basa en el rol que la universidad y la educación superior enfrentan en la actualidad, a partir de los diversos cambios que la sociedad ha sufrido con los avances de la ciencia y la tecnología; lo que ha provocado que la universidad como entidad inmersa en la sociedad deberá ser protagonista de estos cambios y proponer nuevas posibilidades de enfrentarlos y apoderarse de los mismos para ser más competitiva.

Palabras clave: educación superior, innovación, investigación.

ABSTRACT

The present article is based on the role that the university and the higher education faces at the present, from the diverse changes that the society has suffered with the advances of the science and the technology which has provoked that the university as institution immersed in the society will be protagonist of these changes and to propose new possibility of facing them and taking possession in order to be more a competitiveness.

Keywords: education, higher education, innovation, research. 


\section{Introducción}

El avance del desarrollo social va de la mano del conocimiento y de la capacidad de desarrollar la ciencia y la tecnología como roles principales de la universidad actual, con el fin de enfrentar los retos del presente siglo en lo económico y social.

Actualmente, ya no se concibe la universidad como una simple academia transmisora de conocimientos y formación de nuevos profesionales. Las universidades europeas se han planteado una tercera misión de cara al siglo XXI: investigación, desarrollo e innovación.

Esto se inició en las universidades anglosajonas con los planteamientos de Gibbons, Slaughter y Leslie en los años 90, los cuales fueron aceptados por la Unión Europea pues responde a la demanda social del presente siglo.

La universidad debe hacer frente a los retos que tiene con la sociedad; compromiso ineludible de no solamente ser una institución académica, sino también un agente socioeconómico que permita, a través de la investigación, el desarrollo, la innovación, el progreso y el bienestar. Por otra parte, la Organización Mundial del Comercio concluye que se debe liberalizar la educación universitaria como servicio, lo cual quitaría la función social que ejerce. En contraposición, UNESCO en su convención de 1997 (Hurtt, 2000) rechazó este planteamiento. Entonces, nos enfrentamos a dos corrientes de pensamiento; una donde el conocimiento global estará concentrado en círculos de poder y otra, en donde el conocimiento beneficiará a las grandes mayorías en cuanto a su bienestar, progreso y desarrollo. Ante tal situación, la institución universitaria actual no debe menos que reaccionar y replantearse su rol ante la realidad que afronta.

En este artículo se aborda un estudio realizado a partir del rol que ha ejercido la universidad dentro de la educación desde la investigación e innovación; y su influencia con el mercado laboral.

\section{Base teórica}

La educación superior en E1 Salvador ha crecido en los últimos 15 años en cuanto a población estudiantil; según el Ministerio de Educación (MINED) en sus registros estadísticos 20022012, se tuvo una matrícula estudiantil en 2002 de 113,336 estudiantes y para 2012, se registró una matrícula de 169,860 estudiantes. Lo que implica que las universidades están enfrentando varios retos que la misma demanda les exige, su misión de transmisión de conocimientos ha sido una constante desde sus inicios. Es oportuno mencionar el surgimiento de universidades privadas a finales de la década de los 70 debido al conflicto armado en El Salvador que obligó el cierre de la universidad estatal. A pesar de ello, el desarrollo económico y social siguió su curso en muchos países, lo cual ha hecho que la universidad salvadoreña se planteé que su aporte en este rubro debe incrementarse.

Si partimos que la investigación por definición es un proceso que nos permite originar nuevos conocimientos; las universidades salvadoreñas cumplen con esta tarea y por otra parte, si entendemos a la innovación como un avance que surge de la investigación hacia la obtención de nuevos productos; las universidades no desarrollan dicha tarea, se quedan solo con la primera. 
Las universidades desarrollan investigaciones sobre todo de carácter social que han sido para plantear y describir problemas, y en el mejor de los casos, proponer soluciones; en realidad, la escasa vinculación con el sector productivo hace que las universidades trabajen de manera aislada y que sus investigaciones se vuelvan intrascendentes; es por esto que se requiere del asocio públicoprivado para dar un aporte de solución en cuanto a la innovación y al desarrollo; una de las razones es la escasa valoración que el sector productivo empresarial otorga a la calidad de la educación e investigación universitaria, y también la falta de capacidad del mismo sector para absorber el nuevo conocimiento. En general, la universidad salvadoreña tiene como gran desafío orientar sus investigaciones hacia el desarrollo y la innovación donde se puedan obtener propiedad intelectual, patentes e innovaciones que puedan entran al mercado global y den su aporte al desarrollo.

En particular, la única universidad pública de El Salvador hace un gran esfuerzo a pesar de contar con un bajo presupuesto, el más bajo de toda Centroamérica; el cual es el $1.67 \%$ del total del Presupuesto General de la nación; sin embargo, le ha apostado al desarrollo de la ciencia y tecnología: ha creado el Instituto de Ciencias del Mar, el Centro de Investigación en Salud y otros que necesitan fortalecerse, además, de poder incorporar a futuro otras áreas como la biotecnología y la nanotecnología. Las universidades del sector privado han realizado también sus esfuerzos de cara a promover la investigación científica y generar nuevos conocimientos, existen unidades y centros de investigación desde donde las universidades desarrollan tal actividad; no obstante, falta que esa investigación se vincule directamente o aporte al desarrollo económico-social, ya que se necesita de estudios científicos que se conviertan en innovaciones que aporten al bienestar social en general y también una fuerte vinculación con el sector productivo pues este podría maximizar dichas innovaciones.

Los países industrializados (Estados Unidos, Japón, Alemania) son los líderes mundiales en el aspecto de la vinculación entre la universidad $y$ el sector productivo (SP), por tal motivo ellos han sido quienes han tomado decisiones acerca de los mecanismos del mercado mundial, en las relaciones internacionales, y aún en asuntos de política interna de otros países; en cambio, los países menos desarrollados han tenido que reforzar su sistema científico tecnológico y están promoviendo la vinculación entre la universidad y el SP, con la intención de prepararse para responder a los desafíos de la sociedad moderna y para integrarse a la globalización de la economía mundial.

En los países industrializados se han aprovechado al máximo "la capacidad y la tradición de sus pueblos en cuanto a su creatividad y su cultura; y se han preocupado de la preparación de su población en el terreno educativo, ligada al trabajo y a los avances de la ciencia y la tecnología, lo que ha propiciado la vinculación entre la universidad y el SP, como un elemento que contribuye a la formación de sus estudiantes, así como al desarrollo económico y social” (Acuña,1993).

En cambio, los países no industrializados o en vías de desarrollo han descuidado su tradición, su cultura, el desarrollo educativo, la ciencia y la tecnología, lo cual ha contribuido a generar elevados niveles de desigualdad social y 
económica, lo que se refleja en una exagerada pobreza en amplios sectores de su población.

Los datos de los países industrializados demuestran que la eficacia y calidad de la universidad dependen fundamentalmente de las articulaciones que han establecido con la sociedad; pero en América Latina la universidad y el SP han evitado esta relación, debido a que la universidad tiene temor de desvirtuar sus funciones sustantivas y el SP presenta marcados prejuicios respecto al carácter académico de los universitarios, a los cuales ubican alejados de la realidad de la producción. Sin embargo, en los últimos años de la década de los ochenta y principios de los noventa se ha dado en América Latina una mayor importancia a esta vinculación, propiciada por la nueva situación internacional; particularmente, en lo concerniente a la globalización económica, que exige un elevado nivel de eficiencia de las instituciones de educación superior (IES) para lo cual la vinculación de la universidad con el SP representa una opción.

Gibbons (1998) menciona que en las próximas décadas las unidades de producción de bienes y servicios serán más automatizadas y requerirán trabajadores calificados, lo que llevará a un desplazamiento paulatino de la mano de obra no calificada. Para enfrentar el problema del desempleo, la recalificación de la fuerza de trabajo jugará un rol de primera importancia, en el cual las IES, junto a otras instituciones de capacitación laboral, ocuparán un lugar estratégico.

De acuerdo con Gibbons (1998), en las tendencias del empleo para el año 2020 el 90\% de la mano de obra estará ocupada en la micro, pequeña y mediana empresa, tanto en el sector industrial como en el de servicios. Las unidades de producción de bienes y servicios serán más automatizadas y requerirán trabajadores calificados, lo que llevará a un desplazamiento paulatino de la mano de obra no calificada. Para enfrentar el problema del desempleo, la recalificación de la fuerza de trabajo jugará un rol importante, en el cual las IES, junto a otras instituciones de capacitación laboral, ocuparán un lugar estratégico ya que a futuro se espera que la vinculación entre educación y sector productivo se inscriba en el marco de la complementación de las políticas y programas de desarrollo social y económico, en los sectores agropecuario, industrial y de servicios;, y las políticas y programas de desarrollo educativo.

Por lo que Gibbons señala algunas de las posibles características que se requieran del empleo y del trabajo:

- Contracción del empleo en el sector público y crecimiento relativo en el sector privado.

- Disminución de las oportunidades de empleo en grandes empresas.

- Aumento de oportunidades en el empleo no estructurado y de la economía informal.

- Ritmo creciente de cambio en la estructura de puestos y la exigencia de una mayor cualificación en casi cualquier ocupación.

- Pérdida de estabilidad y seguridad en el trabajo y creciente "informalización” en las relaciones entre empleador y empleado. 
- Tendencia a la racionalización y disminución de los puestos que requieren bajos niveles de educación.

- Demanda creciente de conocimientos de informática, idiomas extranjeros y capacidad para el manejo de las nuevas tecnologías de la información y la comunicación.

- Aumento en las funciones laborales que exigen altos niveles de conocimiento en diversas esferas.

En este contexto, Acuña (1993) señala que esta vinculación "consiste en una acción concertada entre ambas corporaciones, en las que el Estado cumple un papel importante, éste participa como elemento integrador del proceso de vinculación", de tal manera, que los principales actores de la vinculación son la universidad, el SP y el Estado, cada uno de los cuales deberá desempeñar un papel exclusivo.

El objetivo de la vinculación, desde el punto de vista de la universidad, debe entenderse como el mecanismo que ayuda a elevar la calidad de la investigación y de la docencia universitarias y a lograr su mejor integración con las necesidades sociales. Desde el punto de vista de la empresa, la vinculación tiene como objetivo elevar la competitividad en el mercado, a través del incremento de la productividad de procesos que aseguren una mayor producción de bienes y servicios por medio de la tecnología transferida desde la universidad, que corresponde en la mayoría de los casos a innovaciones tecnológicas que aseguran productos de mejor calidad y de menores costos”, Acuña (1993).
Por su parte, Reséndez (1998) hace referencia a que las universidades "son organizaciones especializadas que la sociedad requiere principalmente para dotar de ciertas capacidades intelectuales y actividades socialmente destacables a una fracción más o menos amplia de sus jóvenes, antes de que éstos asuman responsabilidades en la vida social y en el trabajo"; por lo tanto, a las universidades les corresponde la responsabilidad de preparar a sus egresados para que asuman desde los primeros años de su formación profesional, la responsabilidad de enfrentarse al ámbito laboral con los elementos necesarios y suficientes para incorporarse a un mercado de trabajo o bien para crear sus propias fuentes de empleo, y esto solo será posible si no permanecen aisladas del ambiente laboral en el que la mayoría de sus egresados van a trabajar.

En este apartado, las empresas, al contratar profesionales que carecen de las habilidades requeridas en el puesto que van a ocupar, tienen reducción en sus niveles de competitividad y gasto en su capacitación. En muchos casos los profesionales carecen de éstas habilidades por el ambiente y el modo en que aprendieron, que frecuentemente son aislados del medio laboral y no por el contenido de los planes y programas de estudio que cursaron en las instituciones educativas.

Por ello, las universidades y las empresas deben mantener entre sí una intercomunicación continua por conveniencia mutua y obtención de beneficios para ambas partes.

Según Reséndez (1998), los propósitos o fines precisos que deben cumplir la vinculación universidad-empresa son, entre otros, los siguientes: 
- Afinar la concordancia de los programas y métodos educativos con las capacidades que las empresas requieren de sus profesionales y técnicos.

- Prever conjuntamente la posible evolución de las necesidades de las empresas y los jóvenes, pues las universidades no educan para hoy, sino para el futuro en que actuarán sus egresados. Tal previsión será más atinada si para ella se conjuga el saber de las empresas y el de las universidades, y no si cada una realiza aisladamente sus actividades.

- Llevar y traer conocimientos y avances técnicos entre universidades y empresas, pues cada una de ellas tiene en estos aspectos, en diferentes momentos y temas, alguna ventaja sobre su contraparte; y por tanto, algo útil que ofrecer para su correspondiente misión.

- Prever las implicaciones de aquellos avances para el futuro de las empresas y de las universidades, e idear juntas modos de enfrentar oportunamente y del mejor modo los cambios previsibles.

- Desarrollar conjuntamente criterios y métodos para evaluar conocimientos y habilidades relevantes para fines laborales y sociales.

- Atenuar el desconcierto o la sorpresa traumática que en los jóvenes produce el paso brusco de la escuela al trabajo.

- Atender al mismo costo las necesidades de actualización y educación continua de los cuadros profesionales y técnicos de las empresas y de los académicos de las universidades, dado que hoy los saberes avanzan muy rápidamente y los saberes que las universidades y las empresas poseen son mutuamente complementarios.

- Resolver problemas de las empresas con el conocimiento científico acumulado en las universidades, mediante la creatividad de los jóvenes a quienes éstas educan.

Por su parte, Villarreal (1995) indica que estos propósitos son:

- Establecer una comunicación más estrecha y frecuente entre las universidades y sus egresados ocupados en las empresas, como canal de retroalimentación para que las universidades conozcan mejor las cambiantes necesidades de las empresas en materia de aprovechamiento del conocimiento tecnológico y, de manera simétrica, para que el personal, educado profesionalmente de las empresas, se entere con igual oportunidad acerca del conocimiento tecnológico disponible en las universidades.

- Promover la investigación tecnológica por contrato entre las empresas y las universidades.

Los fines anteriores y posiblemente algunos más deben considerarse cuando se habla de la vinculación entre las universidades y las empresas.

\section{Metodología}

Se trabajó con una metodología inductiva con el fin de explorar el objeto de estudio planteado por medio de las técnicas que dicha metodología propone. En esta investigación 
se optó por la técnica de grupos focales para conocer sus opiniones y actitudes sobre el problema estudiado, esta técnica fue dirigida a autoridades universitarias (consejos directivos, decanos, administradores académicos) de 14 universidades aglutinadas en la Asociación de Universidades Privadas de El Salvador (AUPRIDES) en la cual participaron 35 sujetos con quienes se indagó sobre los procesos de investigación e innovación que cada institución desarrolla. También se recurrió a la indagación bibliográfica por medio de referencias como libros, revistas y repositorios digitales en los cuales se investigó la evolución de la educación superior en El Salvador desde los años 80 y su rol desde la investigación e innovación, en relación con el mercado laboral. Además, se desarrollaron entrevistas semiestructuradas con representantes de las gremiales empresariales como la Cámara de Comercio de El Salvador y la Asociación Salvadoreña de Industriales, en las cuales participaron 55 personas con quienes se indagó sobre la importancia del sector productivo y su vinculación con las universidades hacia el desarrollo, por medio de la investigación e innovación; además de indagar sobre los perfiles laborales que las empresas demandan para reclutar personal.

\section{Resultados}

\section{El papel del sector privado}

La empresa privada es la receptora directa de los profesionales egresados de las universidades, aparentemente solo este interés les vinculan; sin embargo, si vemos al sector productivo como tal, promotor del desarrollo, necesita de la academia y la academia necesita del sector privado. Juntos pueden dar un gran aporte a al desarrollo económico-social. La Universidad como generadora de la investigación puede iniciar innovaciones que al sector privado le interese producir y comercializar.

Según expresaron varios de los representantes de las gremiales empresariales, se necesita que las universidades cambien su "estilo de formación" pues se enfrentan a un profesional con mucho conocimiento pero con poca preparación práctica. También hacen énfasis en el hecho de que un profesional ya formado no va a llegar a aprender a la empresa.

Como se ve; si bien la misión de las instituciones de educación superior y de las empresas es diferente, existen puntos de convergencia entre ambas que implican una colaboración mutua que tenga como resultado la satisfacción de necesidades de las dos partes.

En cuanto a sus expectativas con respecto al perfil laboral, las empresas exigen un profesional que esté preparado, que sea práctico y capaz de trabajar con iniciativa y resolver problemas; además de la capacidad hacia el trabajo autónomo y en equipo.

Este sector es un eje fundamental en la educación ya que recibe a los profesionales egresados de las universidades y como exploramos en esta investigación es poca la vinculación que hay con dicho sector, por lo que se sugiere establecer alianzas, convenios, proyectos en conjunto que fortalezcan dicha vinculación; de lo contrario, las universidades seguirán graduando profesionales sin saber cuál es la necesidad real del sector productivo en cuanto sus demandas, lo que 
afectará la formación del futuro profesional pues no estará preparado para insertarse fácilmente al mercado laboral. Por otra parte, se perfila cambiar la filosofía de formación pues se ha tenido durante décadas una educación para el trabajo. Hoy en día se ha introducido un nuevo concepto de formación hacia el emprendimiento; es decir, que el futuro profesional se prepare para generar empleo.

\section{El papel de las universidades}

Otros de los hallazgos más importantes se refiere a las carreras que ofertan las universidades, ya que se vinculan más a lo que demanda el estudiante y no a lo que demanda el mercado laboral. Por ejemplo, de cada 100 graduados universitarios cada año, la mitad son abogados, administradores o profesores. La Asociación de Universidades Privadas de El Salvador ha creado convenios con la Asociación de Industriales de El Salvador, con el fin de estructurar el perfil académico que exige la productividad del país. Para el director de AUPRIDES, Reynaldo López, el perfil del graduado está claro, el joven debe tener capacidad profesional en la disciplina estudiada y compromiso ciudadano respecto a ser un ente activo que incida en el desarrollo de la nación. Ante las discrepancias educativas y laborales, el Ministerio de Educación está ampliando las perspectivas en el nivel de formación superior que encumbre la sociedad tecnológica en el país. A través del Viceministerio de Ciencia y Tecnología, los programas educativos se pretenden enrumbar a la tecnología y lo científico. Por otra parte, algunos de los funcionarios universitarios entrevistados indicaron que el país necesita formar ingenieros y técnicos para solucionar problemas prácticos.
También se encontró que la demanda por las carreras de las Tecnologías de la Información se ha incrementado a partir del 2011, año en el cual se registró una inscripción de 14,000 estudiantes.

Otro de los hallazgos importantes que se registraron fue que existen pocos proyectos que vinculan al sector privado con la academia; generalmente se basan solo en colaborar con una empresa a través de un servicio social, donde el estudiante cumple con ciertas horas como requisito para poder graduarse, dichos proyectos no vinculan directamente al estudiante con la empresa, en cuanto a aportar lo aprendido en la academia; sino más bien a cumplir tareas administrativas que no tienen relación con lo que el futuro profesional estudia. Sin embargo, existen también excepciones como la Universidad Don Bosco donde se ha implementado un modelo de formación en Técnico en Aeronáutica junto con la aerolínea AVIANCA, en el cual el graduado de esta carrera puede lograr con éxito un empleo en esta empresa. Otro ejemplo de la necesidad de este vínculo con el mercado laboral y la academia es la Incubadora de Empresas GERMINA, impulsada por la Universidad Francisco Gavidia desde 2009 y donde todo aquel profesional que desarrolle una idea innovadora hacia la productividad que requiere el país puede convertir dicha idea en una empresa.

\section{Conclusiones}

La universidad como una entidad que basa su accionar en el desarrollo de la sociedad deber plantearse varios retos entre estos se encuentra la necesidad de que su rol ha cambiado y que el mismo debe estar sujeto a lo que la realidad demande. Nos encontramos en una sociedad 
del conocimiento y en un mundo globalizado que exige un profesional altamente competente que sepa solucionar problemas que demanda el mercado actual.

Para lo anterior, se recomienda al concluir este estudio que la vinculación educación y mundo laboral debe basarse en tres ejes principales:

1. Sistema de enseñanza: un cambio especial en la metodología que las universidades están aplicando tiene que partir de una aproximación teórica a un enfoque práctico, es una queja constante que quien se gradúa sabe mucho pero no pueden aplicar lo que ha aprendido.

2. Relación con el sector industrial y productivo en el mercado laboral. Hay tan pocos acuerdos y alianzas entre este sector y las universidades, en consecuencia, el sector productivo exige un tipo de profesional pero las universidades y sus profesionales no responden a esta demanda.

3. Desarrollo de proyectos pertinentes y la investigación científica. La mayoría de las universidades se desarrolla sobre todo en la enseñanza y la educación, pero pocas actividades se centran en la investigación científica que se presenta muy débil. No se conectan con el fin de innovar y proponer nuevas ideas o proyectos a los problemas urgentes de nuestro país y del mercado laboral.

Por otra parte, las universidades salvadoreñas han comenzado a plantear el emprendimiento como alternativa hacia sus futuros profesionales, ante la necesidad de enfrentar los problemas económicos que se han suscitado recientemente, ya que han tomado en cuenta que su formación ha ido dirigida a que sus egresados puedan desarrollarse en un empleo y han visto que el mercado laboral no absorbe fácilmente a los nuevos profesionales. En ese sentido, deben esforzarse en formar también a futuros emprendedores que con sus capacidades puedan generar empleos, a través de la creación de nuevas empresas; esto conlleva también a replantearse los planes de estudio hacia esta área.

Otro elemento importante que se concluye, de acuerdo con lo expresado por las personas entrevistadas del sector productivo, es la necesidad de que los profesionales tengan dominio del idioma inglés y las tecnologías de la información y comunicación (TIC) ya que con los tratados de libre comercio, las empresas establecen relaciones comerciales con otros países donde el inglés es idioma de mediación. A su vez, la universidad salvadoreña sigue graduando profesionales de carreras que podemos llamar tradicionales como Derecho, Administración de Empresas, Contaduría, más por lo que el estudiante demanda y no por la necesidad del país; poco se ha hecho por impulsar las carreras técnicas y tecnológicas, aun cuando el Gobierno creó en 2005 los Megatec (Modelo Educativo Gradual de Aprendizaje Técnico y Tecnológico) en zonas del interior del país donde se perfilan polos de desarrollo, en 2006 la matrícula de jóvenes en carreras técnicas ascendía a 11,855 y cuatro años después, incrementó a apenas a 17,952; es decir, seis mil más. Este número corresponde a un $10 \%$ u $11 \%$ de la población estudiantil en el nivel superior. Lo cual significa que las carreras técnicas son poco atractivas para los estudiantes; y es poco lo que se hace para promover la preparación técnica. 
En general, las universidades deben replantear sus acciones hacia la investigación la cual debe llevar a propiciar la innovación y atraer al sector productivo, con el fin que estos dos pilares: investigación e innovación generen mejores empleos y desarrollo para el país.

\section{Referencias bibliográficas}

Acuña, P. (1993). Vinculación universidad sector productivo, en Revista de la Educación Superior, n. ${ }^{\circ} 87$, julio - septiembre 1993, pp. 125150. México: Editorial ANUIES.

Arredondo Galván, V. et al. (1990) La educación superior y su relación con el sector productivo. Problemas de formación de recursos humanos para el desarrollo tecnológico y alternativas de solución. En Revista de la Educación Superior, n. ${ }^{\circ}$ 76, octubre - diciembre 1990, pp. 103-130. México: Editorial ANUIES.
Hernández Rodríguez, R. (2006). El vinculo Universidad Empresa y su relación con la competitividad de los paises. Alemani: Universitat Flensburg.

Machuca, J. (2006). Diagnóstico nacional sobre las políticas y el estado de la investigación científica en la Universidad de El Salvador $\mathrm{y}$ las universidades privadas. REESCA Red de Estudios de Educación Superior de Centroamérica. 